\title{
Fuzzy logic control implementation in a PVC extruder for temperature control in a cable manufacturing industry
}

\author{
Mahesh Kr. Nandwana ${ }^{1}$, Niroj Pokhrel ${ }^{2}$, S. N. Singh ${ }^{2}$ and J. Kumar ${ }^{2}$ \\ ${ }^{1}$ Dept. of Electrical \& Electronics Engineering; ${ }^{2}$ Dept. of Electronics \& Communication Engineering \\ National Institute of Technology, Jamshedpur - 831014, India \\ mahesh.nandwana@gmail.com; nirojpokhrel@gmail.com
}

\begin{abstract}
A number of steps are involved in manufacturing process of an insulated wire. One of the most significant steps of all is controlling the temperature of the poly vinyl chloride (PVC) material which we are going to control using fuzzy logic. The required temperature is set in accordance to the grade of the PVC material available, with no need of manual surveillance. The purpose of this paper is to implement fuzzy logic in wire manufacturing and thus make it autonomous.
\end{abstract}

Keywords: Fuzzy logic, PVC, PVC extruder, fuzzification, defuzzification.

Introduction

The wire and PVC material undergoes several steps before insulated wire is manufactured. The PVC material available initially has large moisture content which has adverse effects in the wire manufacturing process. Mostly, the oven is used for drying process and the temperature control is often done by manually. This imposes the greatest problem as the slightest negligence can result in the burning out of the PVC material changing its colour and thus making it useless. After the moisture is driven out, the PVC material is fed into extruder machine. In the hopper, again the temperature has to be maintained so that the raw material gets melted forming paste which goes to the spindle and covers the wire thus insulating it. We are going to use fuzzy logic for controlling all these process thus overcoming all the hurdles faced by the manual control. Furthermore, the provision of auto cut system when the PVC material has reached the optimum temperature helps to conserve power. Fig. 1 shows the wire-extruder machine.

Fuzzy implementation

The fuzzy implementation can be used in the wire manufacturing industry for the automatic temperature regulation in various steps. Depending on the quantity of the moisture content the PVC material is of different grade. In our system design, the grade of the PVC material is fed in. According to the grade our fuzzy based system sets the temperature to drive out the moisture content through oven. The system through the feedback component monitors the prevailing temperature all the time. Keeping the track of the difference between the set temperature and the prevailing temperature, the fuzzy logic controls the temperature of the system (Kahipara \& Bhatt, 2007).

Fuzzy inference scheme-I (FIS-I)

The fuzzy based system designed will set the temperature to make the PVC material moisture free.

\section{Input scaling and fuzzification}

After doing several researches about the wire manufacturing process by visiting the wire manufacturing industries (Incab Industries, 2000), interacting with the professional handling the process and studying the literature (Berkan \& Trubatch, 2000; Bhanot, 2008), finally we came across the fact that the classification of PVC grade available is ambiguous. Thus, we have decided to give total of 20 points to five different grades and use fuzzy logic to remove the ambiguity prevailed in the classification due to lack of distinct category. The grade point is our input and thus is going to determine the set temperature for the removal of moisture from the PVC raw material. The set temperature varies from $60-180^{\circ} \mathrm{C}$ depending upon the input point of grade. In Fig. 2, P is the highest quality of raw PVC material and $T$ is the lowest quality.

$\mathrm{T}=[4,8] ; \mathrm{S}=[4,12] ; \mathrm{R}=[8,16] ; \mathrm{Q}=[12,20] ; \mathrm{P}=[16$, 20].

\section{Output scaling and fuzzification}

According to the grade of raw PVC material, fuzzy based system decides the set point temperature of PVC. The set temperature has been classified into the following classes according to the input which are as follows:

VS: Very small; S: Small; M: Medium; L: Large; VL: Very large; VS = [60, 100]; $S=[60,120] ; M=[100,140]$;

$\mathrm{L}=[120,180] ; \mathrm{VL}=[140,180]$.

Inference rules for F/S-I

After taking an input point grade from the operator, the following rules are processed in FIS-I

The fuzzy module for FIS-I is shown Table 1.

Development of FIS-II

After obtaining the set point temperature from FIS-I, the FIS-II maintains the set temperature of PVC material in the oven. The FIS-II computes the error between the set temperatures obtained from FIS-I and the current temperature of oven and generate the fuzzy logic action to control the oven temperature. The input variable of FIS-II system is the error temperature. Thus FIS-II
Research article

CIndian Society for Education and Environment (iSee)
"Fuzzy logic in wire manufacturing" http://www.indjst.org
Nandwana et al. Indian J.Sci.Technol. 
Indian Journal of Science and Technology

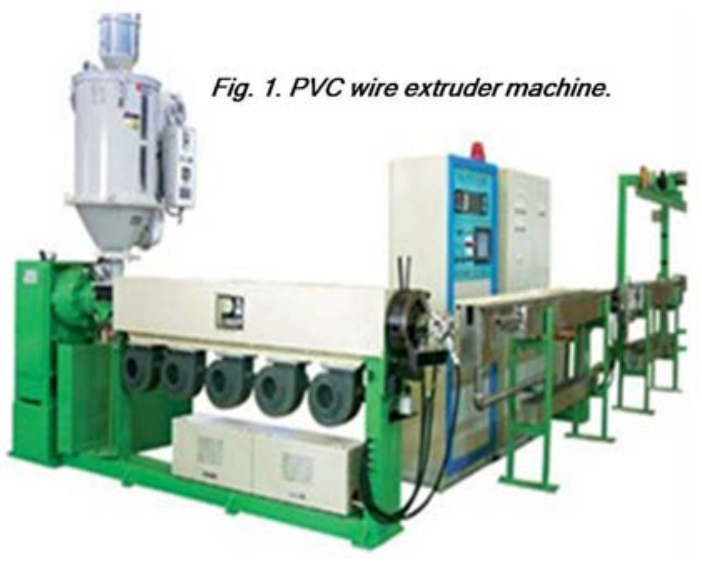

Fig. 3. Membership functions of set temperature.

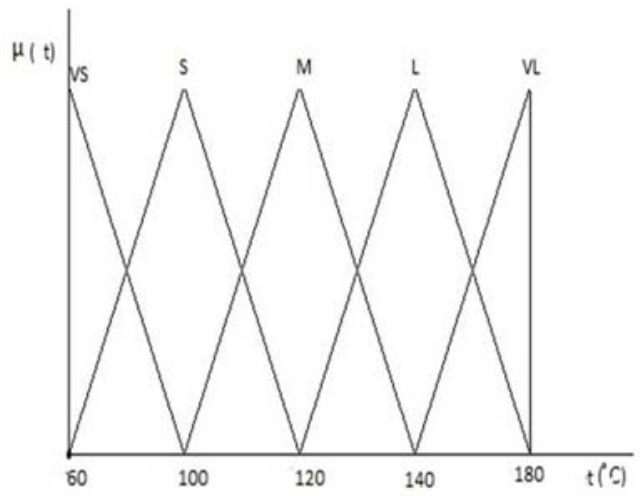

Fig. 5. Membership function of error temperature.

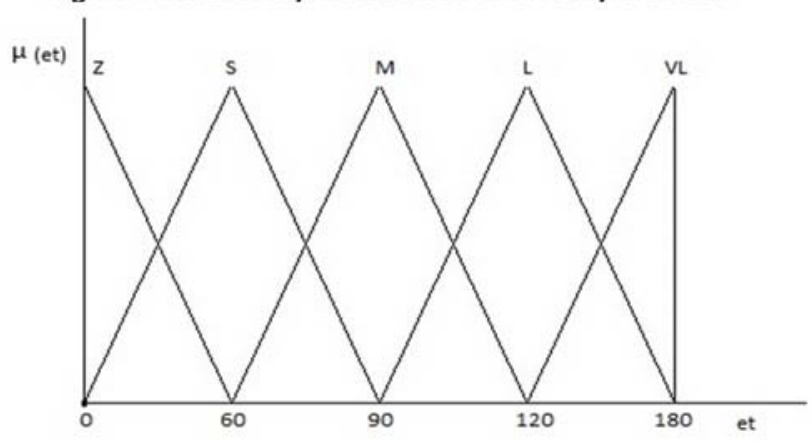

Fig. 7. Fuzzy module for FIS-II.

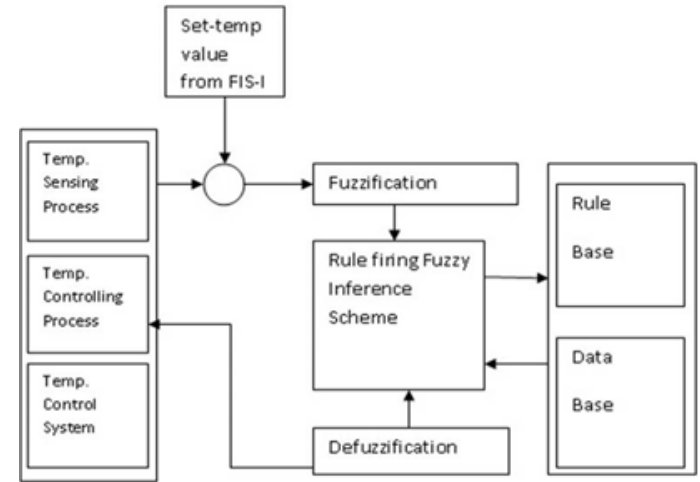

Vol. 3 No. 7 (July 2010)

ISSN: 0974- 6846

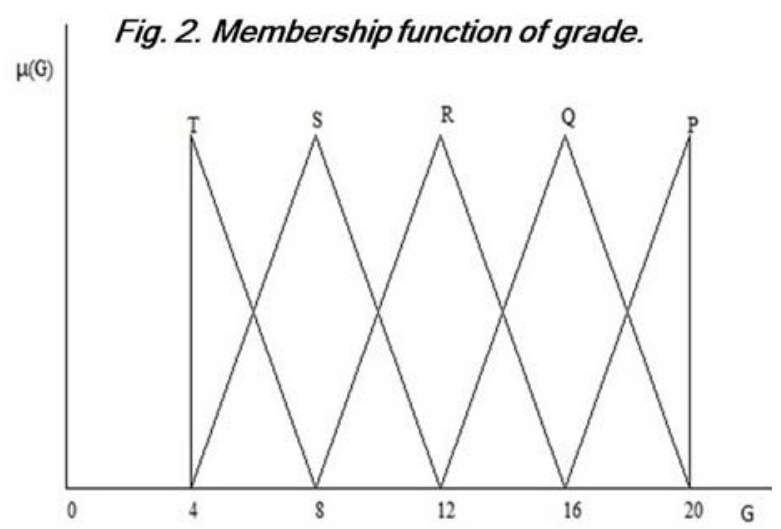

Fig. 4. Fuzzy module for FIS-I.

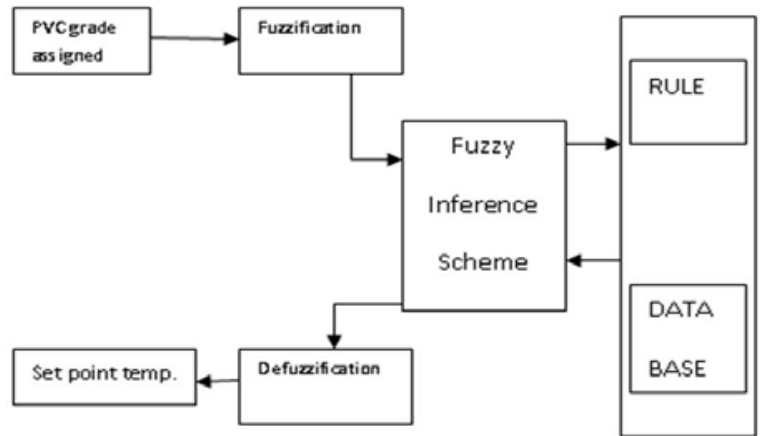

Fig. 6. Membership function of phase angle.

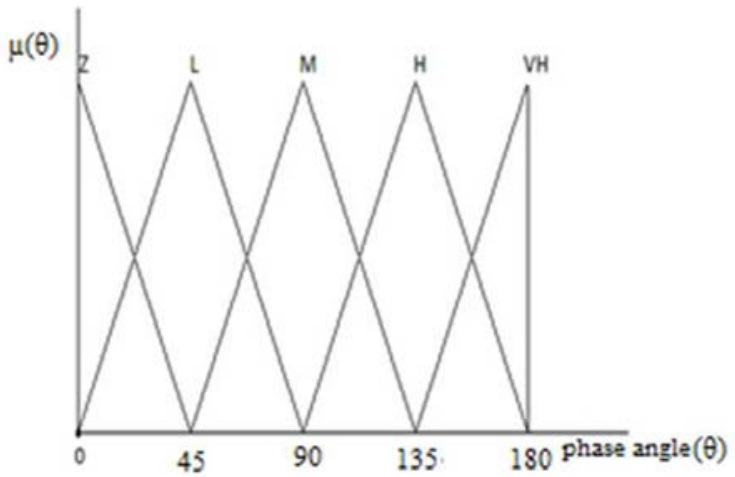

Fig. 8. Computation for set temperature.

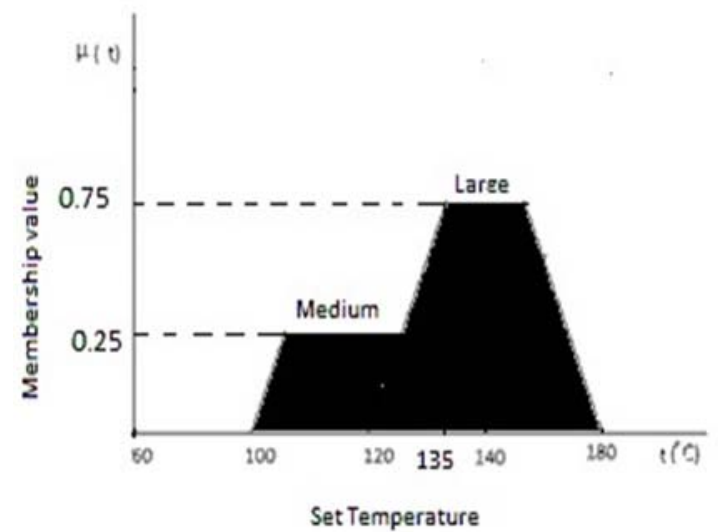

Research article

(C)Indian Society for Education and Environment (iSee)
"Fuzzy logic in wire manufacturing" http://www.indjst.org
Nandwana et al. Indian J.Sci.Technol. 
Fig. 9. Centroid defuzzification for set temperature.

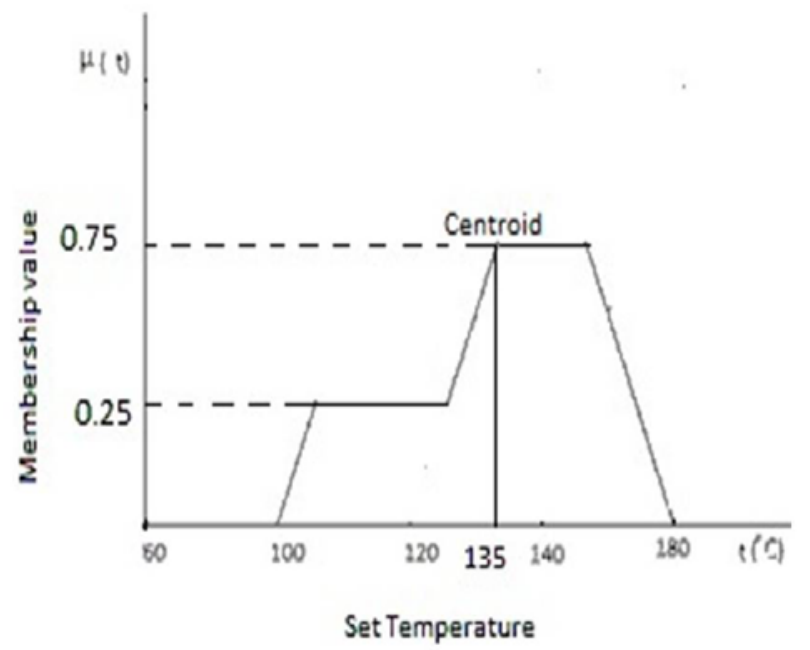

Fig. 10. Computation for phase angle.

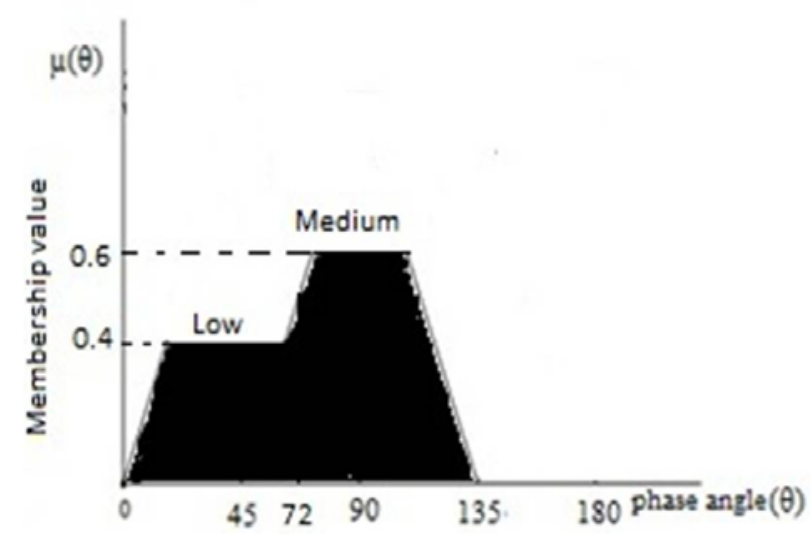

Fig. 11. Centroid defuzzification for phase angle.

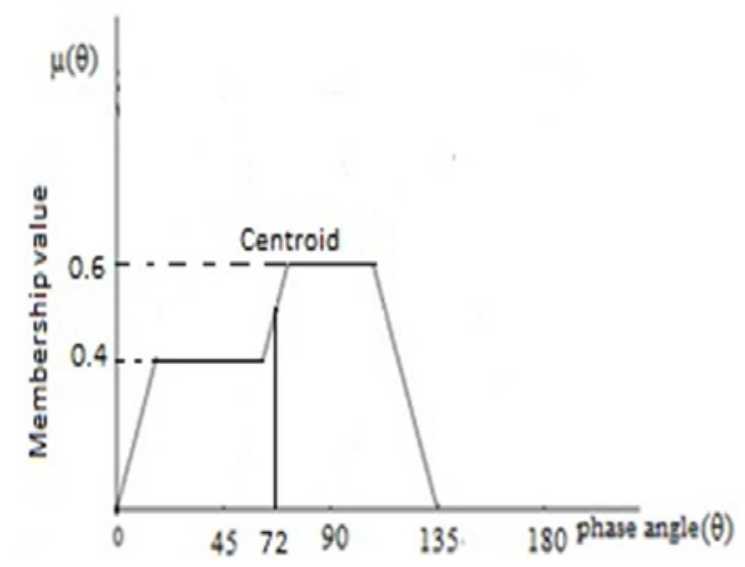

controls the phase angle of triac to control the current which further controls the oven temperature.

Scaling and fuzzification of input

The input variable for FIS-II is the error between the temperature given by FIS-I and current temperature of
PVC in the oven sensed by temperature control system. The membership function of error temperature is shown in Fig. 5. The linguistic values are as follows:

Z: Zero; S: Small; M: Medium; L: Large; VL: Very large; Z $=[0,60] ; S=[0,90] ; M=[60,120] ; L=[90,120] ; V L=[120$, $180]$.

\section{Scaling and fuzzification of output}

According to the rule based on FIS-II, the error temperature value is fuzzified by FIS-II and defuzzified phase angle of the triac is generated which controls the current flowing and thus maintains the temperature. The membership function for the defuzzified output phase angle of FIS-II is shown in Fig. 6. The linguistic values are as follows:

Z: Zero; L: Low; M: Medium; H: High; VH: Very high; $Z=[0,45] ; L=[0,90] ; M=[45,135] ; H=[90,180] ; V H=$ $[135,180]$

\section{Inference rules for F/S-II}

After getting the set point temperature from the FIS-I, the following rules are proposed in FIS-II. The fuzzy module for FIS-II is shown in Table 2. When the above fuzzy logic based system is applied in the wire manufacturing process, it checks all the parameters and starts heating-up the raw PVC material in the oven. The temperature of the PVC material fluctuates up and down the set temperature for a while until reaches to set temperature value with some offset then the system stops heating process. If the PVC level in hopper is below particular level then it is assumed that the PVC is moisture free, than it is assumed that the PVC is moisture free, then it is inserted in the hopper using convener belt arrangement up to a particular level. After this, the second phase of preparing PVC paste begins.

\section{Defuzzification}

The centroid method of defuzzification will be used to calculate the crisp output using the following relation:

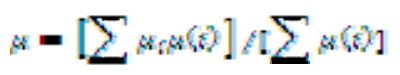

Here, $x_{i}$ represents the action that the $\mathrm{i}^{\text {th }}$ rule would dictate and $k$ (n) represents the truth of $\mathrm{i}^{\text {th }}$ rule.

The grade of PVC material taken was 15 .

From the FIS-I,

$\mu(P)=0 ; \mu(Q)=0.75 ; \mu(R)=0.25 ; \mu(S)=0 ; \mu(T)=0$

The system analyzes and computes the membership function of output variables itself according to the grade of the PVC material. The output graph for membership value of 0.75 for the large membership function and 0.25 for the medium function set temperature has been computed and shown below:

Result of set temperature $=0 / 100 \cup 0.25 / 120 \cup 0.25 / 120$ $\cup$ 0.75/140 U 0.75/140 U 0/180

Graphical solution:

Mathematically,

Crisp output for set temperature= 
Indian Journal of Science and Technology

$\left(0 * 100+0.25 * 120+0.25^{*} 120+0.75^{*} 140+\right.$

$\left.0.75^{*} 140+0^{*} 180\right) /(0+0.25+0.25+0.75+0.75+0)=135^{\circ}$.

The experimental investigation has been taken out at oven temperature of $27^{\circ} \mathrm{C}$.

$\theta$ is the phase angle of the triac.

From the FIS-II,

Error temperature $=105-27=78$

$\mu(Z)=0 ; \mu(S)=0.4 ; \mu(M)=0.6 ; \mu(L)=0 ; \mu(V L)=0$

The output graph for membership value of 0.4 for the small membership function and 0.6 for the medium function phase angle has been computed and shown below:

Result of set temperature $=0 / 0 \cup 0.4 / 45 \cup 0.4 / 45 \cup 0.6 / 90$ U 0.6/90 U 0/135

Graphical solution:

Mathematically,

Crisp output for phase angle = $(0 * 0+0.4 * 45+0.4 * 45+0.6 * 90+0.6 * 90+0 * 135) /(0+0.4+0.4+0$ $.6+0.6+0)=72^{\circ}$.

\section{Conclusion}

Fuzzy logic is suitable for complex, ill-defined, nonlinear process where human experience has edge over mathematical tools. Thus, the application of the fuzzy logic can enhance the wire manufacturing process to be more automated and error free. Furthermore it helps to conserve power and prevent wastage of material.

\section{References}

1. Berkan RC and Trubatch SL (2000) Fuzzy systems design principles, IEEE press, $1^{\text {st }} \mathrm{Ed}$.

2. Bhanot $S$ (2008) Process control: principles and applications, Oxford Univ. Press, Ch. 18.

3. Incab Industries (2000) Newsletter. Jamshedpur, India

4. Kahipara HT and Bhatt TV (2007) Designing an intelligent washing machine using fuzzy logic. Int. Conf. on Trends in Intelligent Electronic Sys. 2, 758761.
Vol. 3 No. 7 (July 2010)

ISSN: 0974- 6846

Table 1. The fuzzy module for FIS-I

\begin{tabular}{|c|c|}
\hline $\begin{array}{l}\text { Fuzzy } \\
\text { function } \\
\text { (FIS-I) }\end{array}$ & Rule \\
\hline$\mu(P)$ & $\begin{array}{c}1 ; \text { if } \mathrm{P}=20 \\
(\mathrm{x}-16) / 4 ; \text { if } 16<\mathrm{P}<20 \\
0 ; \text { if } \mathrm{P}<16\end{array}$ \\
\hline$\mu(Q)$ & $\begin{array}{c}(20-x) / 4 ; \text { if } 16<Q<20 \\
1 ; \text { if } Q=16 \\
(x-12) / 4 ; \text { if } 12<Q<16 \\
0 ; \text { if } Q<12\end{array}$ \\
\hline$\mu(R)$ & $\begin{array}{c}(16-\mathrm{x}) / 4 ; \text { if } 12<\mathrm{R}<16 \\
1 ; \text { if } \mathrm{R}=12 \\
(\mathrm{x}-8) / 4 ; \text { if } 8<\mathrm{R}<12 \\
0 ; \text { if } \mathrm{R}<8\end{array}$ \\
\hline$\mu(S)$ & $\begin{array}{c}(12-\mathrm{x}) / 4 ; \text { if } 8<\mathrm{S}<12 \\
1 ; \text { if } \mathrm{S}=8 \\
(\mathrm{x}-4) / 4 ; \text { if } 4<\mathrm{S}<8 \\
0 ; \text { if } \mathrm{S}<4\end{array}$ \\
\hline$\mu(T)$ & $\begin{array}{c}(8-x) / 4 ; \text { if } 4<T<8 \\
1 ; \text { if } T=4 \\
0 ; \text { if } \mathrm{T}<4\end{array}$ \\
\hline
\end{tabular}

Table 2. The fuzzy module for FIS-II

\begin{tabular}{|c|c|}
\hline $\begin{array}{c}\text { Set point } \\
\text { temperature }\end{array}$ & Rule \\
\hline$\mu(Z)$ & $\begin{array}{c}1 ; \text { if } Z=0 \\
(60-y) / 60 ; \text { if } 0<Z<60 \\
0 ; \text { if } Z=60\end{array}$ \\
\hline$\mu(S)$ & $\begin{array}{c}0 ; \text { if } S=0 \\
(y-0) / 60 ; \text { if } 0<S<60 \\
1 ; \text { if } S=60 \\
(90-y) / 30 ; \text { if } 60<S<90\end{array}$ \\
\hline$\mu(\mathrm{M})$ & $\begin{array}{c}0 ; \text { if } \mathrm{M}<60 \\
(\mathrm{y}-60) / 30 ; \text { if } 60<\mathrm{M}<90 \\
1 ; \text { if } \mathrm{M}=90 \\
(120-\mathrm{y}) / 30 ; \text { if } 90<\mathrm{M}<120\end{array}$ \\
\hline$\mu(L)$ & $\begin{array}{c}0 ; \text { if } \mathrm{L}<90 \\
(\mathrm{y}-90) / 30 ; \text { if } 90<\mathrm{L}<120 \\
1 ; \text { if } \mathrm{L}=120 \\
(180-\mathrm{y}) / 60 ; \text { if } 120<\mathrm{L} 180\end{array}$ \\
\hline$\mu(\mathrm{VL})$ & $\begin{array}{c}0 ; \text { if } V L<120 \\
(y-120) / 60 ; \text { if } 120<V L<180 \\
1 ; \text { if } V L=180\end{array}$ \\
\hline
\end{tabular}

Nandwana et al. Indian J.Sci.Technol. 